\title{
Pancreatoblastoma - A Rare Pediatric Pancreatic Tumor.
}

\author{
Dr Vikas Fotedar ${ }^{1}$, Dr Rajeev K Seam ${ }^{2}$, Dr Manoj K Gupta ${ }^{3}$. \\ ${ }^{I}$ Senior Resident, ${ }^{2,3}$ Professor, Deptt. of Radiotherapy, IGMC, Shimla
}

\begin{abstract}
A 12-year-old male child, who was previously well, complained of pain and lump in abdomen. Physical and radiological investigations revealed a mass in pancreas. Histopathological examination of the resected specimen showed pancreatoblastoma. Pancreatoblastoma is an unusual malignant tumour seen in infants and children although rare cases have also been reported in adults. They are clinicopathologically distinct from adult pancreatic ductal carcinoma. The histogenesis, clinical features and treatment options are discussed along with presentation of the case.
\end{abstract}

Keywords: alpha-foetoprotein,pancreatic tumour, pancreatoblastoma ,PLADO

\section{Introduction}

Pancreatoblastoma is a rare distinct neoplasm that is most commonly encountered in infants and young children, although rare cases have been reported in adults. They comprise $0.5-1 \%$ of pancreatic tumors. Pancreatoblastoma was first described by Frantz in 1959. Subsequently, Frable et al in 1971 described the histological and ultrastructural findings and called it as "infantile carcinoma of pancreas". These tumors are considered to be embryonic in origin, based on the histological appearance. Evidence of endocrine component, acinar cells containing zymogen granules and the presence of alpha-foetoprotein, suggests that this neoplasm arises from multipotential stemcells. A potential molecular association between pancreatoblastoma and hepatoblastoma is suggested by the occurrence of both tumors in young patients with Beckwith-Wiedemann syndrome, raising the possibility that genetic events on chromosome $11 \mathrm{p}$ might play a role. The clinical presentations of these tumors are varied. They can present as abdominal pain, abdominal mass, diarrhoea, or upper gastrointestinal bleeding. Most of the time, they are asymptomatic. The presenting features are highly non-specific and this leads to diagnostic dilemmas. The tumor is slightly more frequent in males, with the median age of presentation being five years. Though malignant, these tumors have an indolent course. They can be cured by complete resection alone and in cases of unresectable tumors, incomplete resection and in those with metastatic lesions, radiotherapy or chemotherapy may be given. The prognosis is worse in the presence of synchronous or metachronous metastasis and non-resectable disease at presentation, ${ }^{[1]}$.

\section{Case Report}

A 12-year-old boy presented with pain and swelling in the upper abdomen and had vomitings. The vomitus contained only ingested food particles. Patient also had few episodes of loose stools. Physical examination revealed a slightly tender lump in the epigastrium and left hypochondriac region. The rest of the abdomen was soft and non-tender. Bowel sounds were sluggish. Ultrasonography of the abdomen showed a well-defined heterogeneous mass in the epigastric region measuring $6.4 \times 5.5 \times 2 \mathrm{~cm}$. This mass was situated medial to the spleen and was compressing the stomach. Computed tomography (CT) of abdomen showed a welldefined cystic mass in the region of pancreatic tail.(Fig. $1 \& 2$ ). The contents antigen, serum amylase and alphafoetoprotein were all within normal limits. The patient underwent exploratory laprotomy for resection of the mass in the pancreas. Intra operatively, there was a well-defined encapsulated firm mass arising from the body of pancreas adherent to mesocolon and lesser sac of stomach. The mass was adherent to retroperitoneum and surrounding structures except spleen This cystic mass was resected piecemeal. Grossly, the resected tumor was globular and encapsulated, measuring $7 \times 6 \times 3 \mathrm{~cm}$ and weighing $180 \mathrm{~g}$. The surface was bossellated and the tumour was soft in consistency. Serial cut sections revealed a greyish white solid tumor with central area of haemorrhage and necrosis. Microscopical examination of this resected tumor showed pancreatic tissue and a tumor that was arranged in glandular and acinar pattern with interspersed focal solid areas. In the solid areas, whorled nests of spindle cells and islands of plump epithelioid cells were seen (Fig. 3 \& 4). Extensive tumor necrosis was also present. The tumor cells showed diffuse strong positivity for alpha-foetoprotein .After surgery, the patient was referred to our Deptt. for further management. He was started on PLADO (Cisplatinum \&Doxorubicin) based chemotherapy .He received one cycle of PLADO based chemotherapy and later was lost to follow up. 


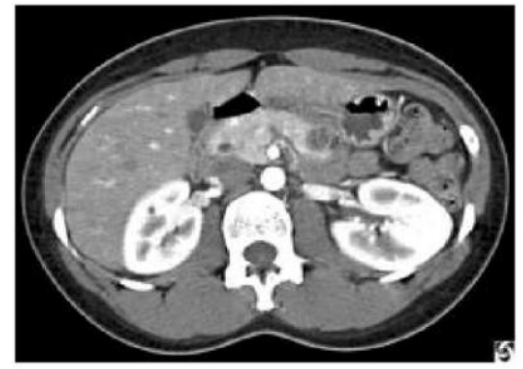

Fig 1: Enhanced axial CT image shows a large well- defined cystic tumour in the the tail of the pancreas.

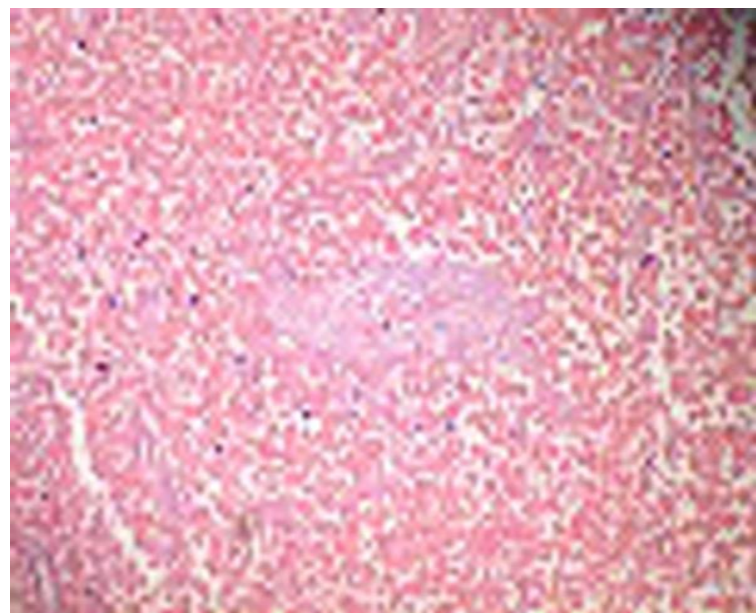

Fig 3: Photomicrograph shows a cellular tumour with uniform epithelial cells arranged in nests and acini.

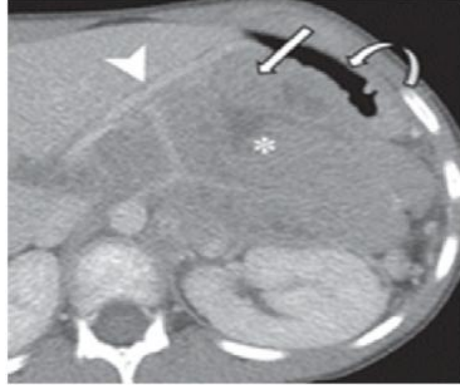

Fig 2: Magnified axial CT image of pancreatoblastoma in the body $\&$ the tail of pancreas.

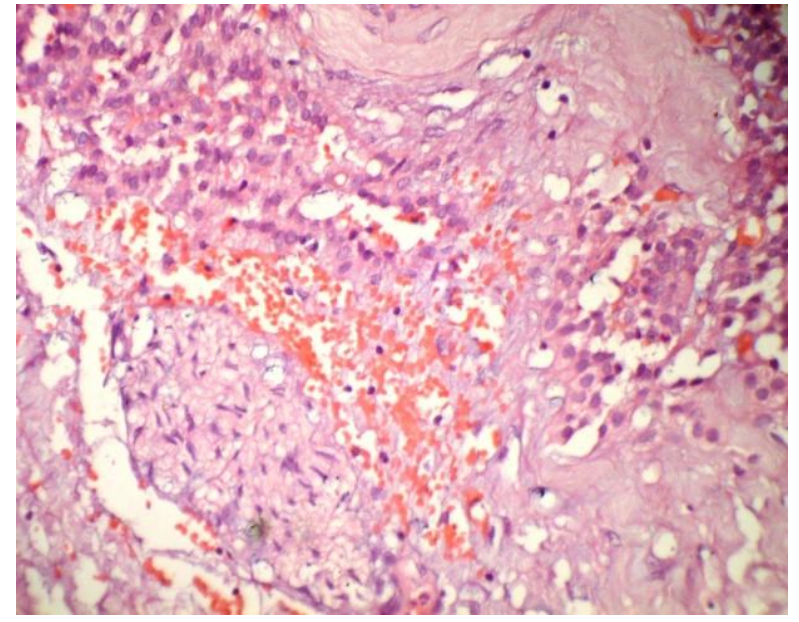

Fig 4: Microsection showing small spindle cell a) acinar arrangement of cells, $b$ ) larger cells with vesicular nuclei.

\section{Discussion}

Pancreatic tumours are rare in children, and pancreatoblastoma comprises only $0.5-1 \%$ percent of pancreatic non-endocrine tumors occurring in children. This tumor is more common in Asians than in the white population. The histogenesis of this tumor is still uncertain. It is said to be hamartomatous or dysembryogenic development of ductal cells of ventral portion of primordial pancreas. Pancreatoblastoma contains pluripotent cells capable of differentiating along the pathway of all three pancreatic cell types. Molecular investigation has disclosed a mosaic paternal $11 \mathrm{p} 15$ uniparental disomy in the tumor cells of pancreatoblastoma, ${ }^{[2] .}$ Recently genetic alterations also have been characterised and the commonest change is allelic loss of $11 \mathrm{p},{ }^{[3]}$. Elevated serum alpha-foetoprotein levels have been reported in up $68 \%$ of cases, ${ }^{[3]}$. The level comes down once the tumor is resected. In our case, the key learning issue is that serum alpha-foetoprotein levels were not elevated but immunohistochemical stain for alpha-foetoprotein was strongly positive in the tumor cells. This kind of behaviour has been reported earlier also ${ }^{[4]}$. This tumor is commonly present in the body and tail of the pancreas, ${ }^{[5]}$ and the prognosis is poorer if the tumor is situated at this site as it is difficult to resect, and hence, there are more chances of recurrence. Tumors measuring up to $25 \times 20 \times 15 \mathrm{~cm}$ and weighing up to $2.5 \mathrm{~kg}$ have been reported. Majority of the tumours are encapsulated, while the rest are partially encapsulated. Encapsulated tumours have a better prognosis. Histopathological features that are readily seen include haemorrhage, capsule formation and necrosis. In our case, the tumor was situated in the body of pancreas, encapsulated and had extensive areas of haemorrhage and necrosis. The tumor can exhibit acinar, endocrine and ductal differentiation ${ }^{[6]}$, with ultrastructural examination of the epithelial cells showing electron dense zymogen granules or small dense neuroendocrine granules ${ }^{[7]}$. The tumor has an indolent course and is amenable for various modes of treatment but surgery is the most optimal treatment. Complete resection of the tumor offers the best prognosis. However, in the presence of metastatic disease, it is of limited value. In these situations where there is suspected or documented metastatic lesions, empirical chemotherapy regimens that include cisplatin and doxorubicin have been used. When the tumor is unresectable and the patient is non-responsive to chemotherapy, radiotherapy is given. Significant shrinkage of the tumour has been reported after treatment with 
radiotherapy. Pancreatoblastoma, though not common, is said to be less aggressive in infants and children compared to adults. Prognosis of this rare tumor is good, when resected completely. Prognosis is poorer, when there is metastasis or when it is inoperable.

On the whole, pancreatoblastoma is regarded to be a curable tumour, hence the clinical diagnosis should be made early. Awareness of the various modes of presentation of this tumor is essential for early detection and proper management.

\section{References}

[1] Dhebri AR, Connor S, Campbell F, et al. Diagnosis, treatment and outcome of pancreatoblastoma. Pancreatology. 2004; 4:441-53.

[2] Pelizzo G, Conoscenti G, Kalache KD, et al. Antenatal manifestation of congenital pancreatoblastoma in a foetus with BeckwithWiedemann syndrome. Prenat Diagn. 2003; 23: 292-4.

[3] Abraham SC, Wu TT, Klimstra DS, et al. Distinctive molecular genetic alterations in sporadic and familial adenomatous polyposisassociated pancreatoblastomas: frequent alterations in the APC/ beta-catenin pathway and chromosome $11 \mathrm{p}$. Am J Pathol. 2001;159:1619-27.

[4] Naik V R, Jaafar H, Leow V M, Bhavaraju V M K. Pancreatoblastoma:a rare tumour accidentally found. Singapore Med J. 2006; 47(3):232-4.

[5] Kohda E, Iseki M, Ikawa H, et al. Pancreatoblastoma. Three original cases and review of the literature. Acta Radiol. 2000; 41:334-7.

[6] Klimstra DS, Wenig BM, Adair CF, et al. Pancreatoblastoma.A clinicopathologic study and review of the literature. Am J Surg Pathol. 1995; 19:1371-89.

[7] Silverman JF, Holbrook CT, Pories WJ, et al. Fine needle aspiration cytology of pancreatoblastoma with immunocytochemical and ultra structural studies. Acta Cytol 1990; 34:632-40.

Legends for illustrations:

Fig 1: Enhanced axial CT image shows a large well-defined cystic tumour in the tail of pancreas.

Fig 2: Magnified axial CT image of pancreatoblastoma in the body and tail of pancreas.

Fig 3: Photomicrograph shows a cellular tumour with uniform epithelial cells arranged in nests and acini.

Fig 4: Microsection showing small spindle cells a) acinar arrangement of cells, b) larger cells with vesicular nuclei. 